\title{
Progress in the Identification of Genetic Factors in Periodontitis
}

\author{
Marja L. Laine • Soren Jepsen • Bruno G. Loos
}

Published online: 18 October 2014

(C) Springer International Publishing AG 2014

\begin{abstract}
The susceptibility to periodontitis is determined by a complex interplay between bacteria, the immune system, and life-style factors, and is mainly regulated by genes. The genetic factors contributing to the pathogenesis of periodontitis are still not fully defined. The aim of the present review is to summarize and discuss the status of replicated candidate gene and genome-wide association studies (GWAS) in aggressive and chronic periodontitis. Currently, 3 susceptibility genes, namely GLT6D1, ANRIL, and COX-2, have repeatedly been associated with periodontitis in cohorts of sufficient size. Most of the associations have been reported in aggressive periodontitis. Single nucleotide polymorphisms (SNPs) within ANRIL and COX-2 seem to also be associated with chronic periodontitis. Further, SNPs within the $I L-10$ gene have shown suggestive associations with aggressive periodontitis, and SNPs within DEFB1 with aggressive and chronic periodontitis. Probably, the identified SNPs are "genetic markers" and not causative variants for aggressive and chronic periodontitis.
\end{abstract}

Keywords Aggressive periodontitis - Chronic periodontitis · Genetics · GWAS · Candidate gene · Periodontology · Periodontitis · Periodontal · Dental · Complex disease · Cardiovascular disease · ANRIL · GLT6D1 · COX-2

\footnotetext{
M. L. Laine · B. G. Loos $(\bowtie)$

Department of Periodontology, Academic Centre for Dentistry Amsterdam (ACTA), University of Amsterdam and VU University, Amsterdam, The Netherlands

e-mail: B.Loos@acta.nl

S. Jepsen

Department of Periodontology, Operative and Preventive Dentistry, Center of Dento-Maxillo-Facial Medicine, Faculty of Medicine, University of Bonn, Bonn, Germany
}

\section{Introduction}

Periodontitis is a complex disease characterized by chronic infection and inflammation of supporting tissues of teeth. In individuals susceptible to periodontitis, there is an imbalance between the host's immune system and the periodontal bacteria. Inflammatory reactions of these individuals to the periodontal bacteria may gradually destroy the periodontium resulting in loss of attachment and alveolar bone. In the most severe cases, the affected teeth will become mobile and may even exfoliate.

There are 2 types of periodontitis: the aggressive and the chronic form. Individuals' susceptibility to both forms of periodontitis is determined by a complex interplay between bacteria, the immune system, and life-style factors such as smoking, stress, and diet, and is mainly regulated by genes (Fig. 1). Furthermore, epigenetic changes of DNA and mutations during lifetime may influence individual's susceptibility to periodontitis. However, the relative contribution of genetic factors to the 2 forms of periodontitis differ (see below).

Evidence for the role of genetics in periodontitis has been gained from population, family, and twin studies [1-4]. Studies in twins and especially in monozygotic twins are a strong and preferred model to study heritability of a disease. Michalowicz et al. [2] studied 110 twin pairs (63 monozygotic and 33 dizygotic twin pairs) with chronic periodontitis and showed that among monozygotic twins a higher concordance of alveolar bone loss patterns was seen than in the dizygotic twins. The heritability estimates for alveolar bone loss among reared-apart monozygotic twins was $38 \%$ [2]. For aggressive periodontitis (actually it was on the early-onset form), there is only 1 study that has reported on the heritability in twins [5]. However, the study consisted of a low number of twins (7 monozygotic and 19 dizygotic twin pairs at age 12-17 years) and, therefore, no clear conclusions could be made on the concordance rate of early-onset periodontitis. Because of a 


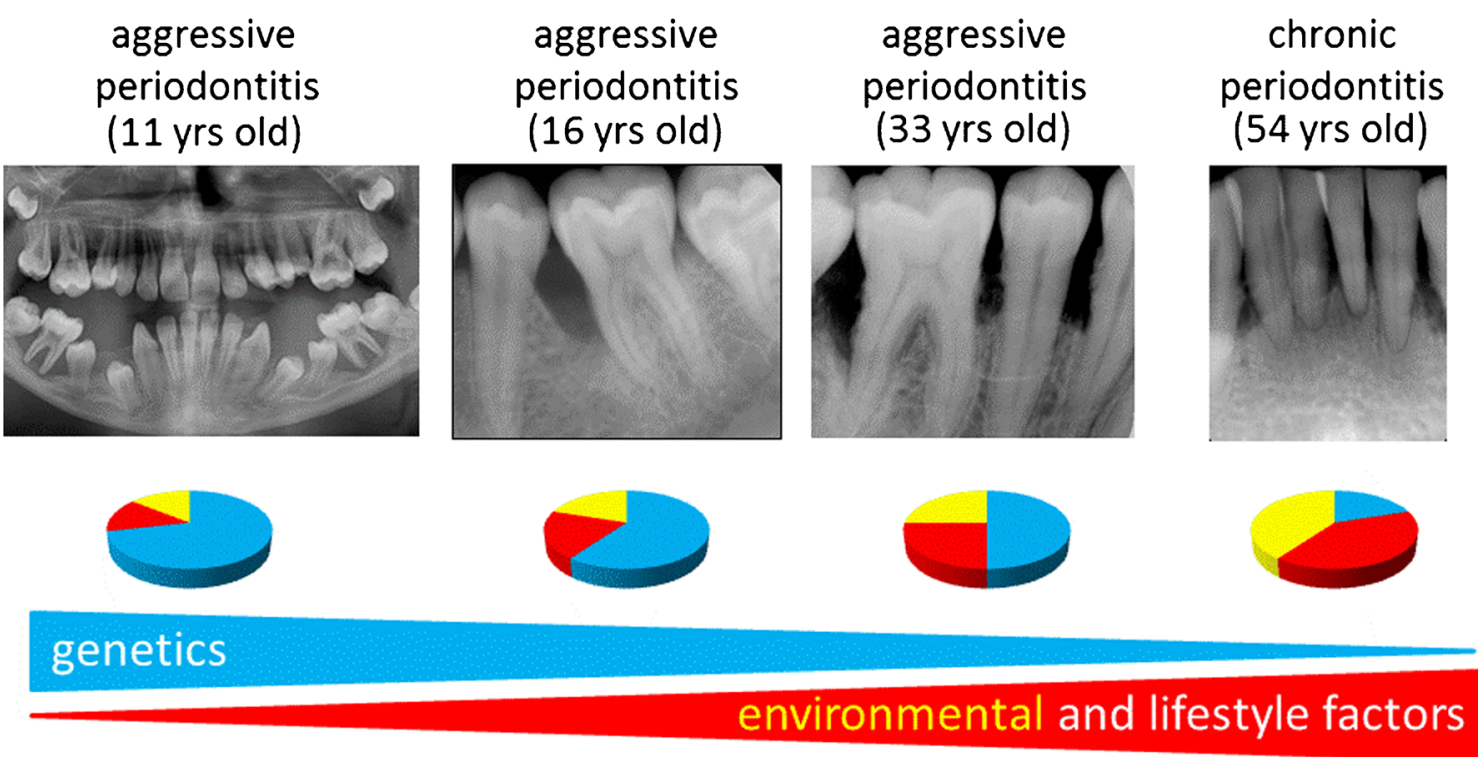

Fig. 1 The estimated proportions of genetics, environmental- and lifestyle factors in aggressive and chronic periodontitis. Blue genetics, red lifestyle factors, yellow environmental factors

relatively low prevalence of aggressive periodontitis in the general population, it is very difficult to identify enough affected twins to provide sufficient statistical power to test the concordance of this disease phenotype. Nevertheless, because of strong familial aggregation, rapid progression and early onset of disease, it is generally accepted that genetic factors play an important role in the disease susceptibility of aggressive periodontitis (Fig. 1) [6, 7]. Similar to other complex inflammatory diseases, it is now clear that the earlier periodontitis manifests itself, the greater the role of genetics factors.

The genetic factors contributing to the pathogenesis of periodontitis are still not fully defined. In the last decades, research on the genetics of periodontitis has focused on identifying specific nucleotide polymorphisms in specific genetic loci as risk factors for aggressive and chronic periodontitis. The present review summarizes recent findings of these genetic studies on candidate genes and genome-wide association studies (GWAS).

In general, genetic studies using a severe disease phenotype are the most useful to identify the genes involved in the disease; for complex diseases, the strongest phenotypes will be affected most by genetic factors (genetic penetrance is high) and will suffer the least from "noise" from environmental and life style factors (more homogeneity in case populations). Therefore, studies employing aggressive periodontitis have less heterogeneity in the study population than chronic periodontitis. The genetic risk loci identified in aggressive periodontitis can then be carried forward for testing as putative risk loci in chronic periodontitis. Notably, the genetic variants identified for periodontitis mostly point to a gene locus, whereas the variants (polymorphisms) mostly are only genetic markers.

\section{Candidate Gene Studies in Periodontitis}

In general, the genetic loci and the known genetic polymorphisms occurring in such loci, are chosen for candidate gene association studies in periodontitis based on their relation with immune responses and/or have previously been associated with other chronic inflammatory diseases such as rheumatoid arthritis, Crohn's disease, type 2 diabetes and cardiovascular diseases (also termed pleiotropy) [8].

The most studied type of gene polymorphism is the single nucleotide polymorphism (SNP). In candidate gene association studies, allele frequencies of selected SNP(s) are determined and compared between a well-defined group of cases and controls. Next to the statistical significance, the results are expressed with an odds ratio (OR). The ORs of common gene polymorphisms associated with complex diseases are typically $\leq 1.5$ [9].

A large amount of candidate gene association studies have been performed in periodontitis with varying and often contradictory results $[10,11]$. Small cohort size resulting in lack of power and the lack of replication have been the major problems in the periodontal genetic studies [12]. Further, most of the candidate gene studies in periodontitis have not captured the complete genetic information of a particular region of interest. In almost all studies, only 1 or a few candidate SNPs instead of complete haplotypes of the genetic locus of interest were genotyped. Furthermore, many studies on genetics in periodontitis are limited because of inadequate phenotype classification of periodontitis and control subjects, as well as not taking into account life style factors such as smoking or the presence of other diseases. 
Aggressive Periodontitis

The associations between aggressive periodontitis and SNPs in the $C D K N 2 B$ antisense RNA 1 (ANRIL) locus can be considered as the best replicated genetic associations in periodontitis to date (Table 1) [13-16]. Interestingly, this genetic locus is a regulatory region and not a protein-encoding gene. The first candidate gene study of the ANRIL gene identified a haplotype block tagged by SNP rs1333048 to be associated with generalized (151 cases vs 736 controls) and localized (137 cases vs 368 controls) aggressive periodontitis in a German population [14]. This association was replicated in a German-Northern Irish population (130 cases vs 339 controls) and in a small Turkish Caucasian study population (89 cases vs 72 controls) $[15,16]$. Importantly, the locus is the top risk locus of coronary artery disease [14]. In a follow-up study by Schaefer et al. [13] the entire $C D K N 2 B A S$ region was genotyped in Dutch (159 cases vs 421 controls) and German (301 cases vs 962 controls) cohorts, but only nominally significant replicated associations were found between aggressive periodontitis and SNPs in the ANRIL locus. It may be concluded that the $A N R I L$ locus is associated with aggressive periodontitis, but the causative SNP still needs to be determined.

Characterization of the molecular function of ANRIL showed, a long-distance regulatory effect on the activity of the CAMTA1/ VAMP3 region, ADIPOR1 and C11ORF10 [20]. Interestingly, the CAMTA1/VAMP3 region has previously shown to be associated with increased periodontal pathogen colonization [20, 21].

The COX-2 SNP rs20417 (haplotype tagged by rs6681231) was first identified in a Taiwanese aggressive periodontitis cohort (85 cases vs 153 controls, (Table 1)) [17] and later also in a Dutch-German aggressive periodontitis cohort (520 cases vs 1043 controls) [18]. So far, these associations have not been replicated in cohorts with same ethnical background.

$\mathrm{COX}-2$ converts arachidonic acid into prostaglandin $\mathrm{H} 2$, which is the precursor of prostaglandin E2 (PGE2). PGE2 mediates pro-inflammatory and anti-inflammatory reactions in many tissues and is also partly responsible for the resorption of the alveolar bone during the pathogenesis of periodontitis. However, whether the identified association is related to altered function of COX-2, and therefore, indirectly could play a role in the pathogenesis of periodontitis is still an open question.

The associations of $I L-10$ SNPs rs61815643 and rs6667202 were first identified in a German Caucasian population of 600 aggressive periodontitis patients and 1441 controls [16]. The associations were further tested in validation cohorts of 164 Dutch aggressive periodontitis patients and 1020 controls, and 105 German-Austrian aggressive periodontitis patients and 482 controls. The genetic region was confirmed in the Dutch validation cohort, but not in the German-Austrian cohort.

The $I L-10$ genetic region seems to be a very interesting region as it has been associated also for example with inflammatory bowel disease and type 1 diabetes [22]. The $I L-10$ gene encodes the anti-inflammatory cytokine interleukine-10 (IL-10). IL-10 can down-regulate the pro-inflammatory immune response of monocytes and macrophages in paracrine and autocrine fashion: IL-10 is produced by monocytes, macrophages, and T cells, and its action results in reduced expression of the pro-inflammatory cytokines such as IL-1 and TNF- $\alpha$.

Schaefer et al. [23] analyzed the candidate gene $D E F B 1$ by fine-mapping, which SNPs were associated with aggressive periodontitis in a cohort of 532 patients and 1472 controls of German-Dutch origin. A suggestive association was found between the DEFB1 SNP rs1047031 and aggressive periodontitis. This association was not replicated in another Dutch-German aggressive periodontitis population, but notably in a chronic periodontitis cohort of German-Dutch origin (805 cases vs 1415 controls) [23]. The rare allele of DEFB1 was predicted to impair a microRNA binding site at the $3^{\prime}$ untranslated region (UTR) of $D E F B 1$. The antimicrobial peptide (human beta-defensin 1) that is encoded by $D E F B 1$ plays a pivotal role in maintaining a healthy status of the mucosal epithelia thus, preventing infection with pathogenic oral bacteria. Again, it still remains unclear how the SNP could influence the pathogenesis of aggressive periodontitis.

\section{Chronic Periodontitis}

Despite the numerous studies on SNPs in chronic periodontitis, only few are currently validated in cohorts of considerable size. The association of $C O X-2$ SNP rs6681231, a haplotype tagging SNP (htSNP) for rs20417 (near-perfect linkage disequilibrium, $\mathrm{r}^{2}>0.95$ ) [18], was initially reported to be associated with chronic periodontitis in Taiwanese chronic periodontitis patients (343 cases vs 153 controls) [17]. Later, Loo et al. [24 and $\mathrm{Li}$ et al. [25] studied the association between chronic periodontitis and the $C O X-2$ SNP rs20417 in Chinese chronic periodontitis patients ( 280 cases vs 250 controls, 122 cases and 532 controls, respectively) and both studies reported on an opposite association in a crude analysis. This confirms that SNPs in this genetic locus are related to periodontitis, but that the real causative $\mathrm{SNP}(\mathrm{s})$ are not yet identified, only tagging SNPs. No association for the $C O X-2$ SNPs has been found in Caucasian chronic periodontitis patients [18].

For the ANRIL SNP rs3217992 a trend was found in chronic periodontitis $(P=0.06)$ [13]. However, this association has not been replicated in another chronic periodontitis cohort to date. The ANRIL SNP rs10811658 was significantly associated with chronic periodontitis in Dutch as well as in German cohorts, also after adjustment for smoking, diabetes, gender, and age. However, after correcting for multiple comparisons the association lost its significance [13].

The $I L-1$ gene polymorphisms are probably the most studied candidate SNPs in chronic periodontitis. Recent systematic reviews and meta-analyses on $I L-1$ gene polymorphisms in Caucasian chronic periodontitis patients and controls, 
Table 1 Repeated associations of specific SNPs in aggressive periodontitis

\begin{tabular}{|c|c|c|c|c|c|}
\hline Gene/SNP & Chromosome & Population & Cases $(N)$ & Controls $(N)$ & Reference \\
\hline$A N R I L$ & $9 \mathrm{p} 21.3$ & & & & \\
\hline \multirow[t]{2}{*}{ rs3217992 } & & German & 301 & 962 & Schaefer et al. (2011) [12] \\
\hline & & Dutch & 159 & 421 & Schaefer et al. (2011) [13] \\
\hline \multirow[t]{5}{*}{ rs 1333048} & & German & 151 & 736 & Schaefer et al. (2009) [14] \\
\hline & & German $^{\mathrm{b}}$ & 137 & 368 & Schaefer et al. (2009) [14] \\
\hline & & German-Northern Irish & 130 & 339 & Ernst et al. (2010) [15] \\
\hline & & Turkish & 89 & 72 & Schaefer et al. (2013) [16] \\
\hline & & German & 594 & 1441 & Schaefer et al. (2013) [16] \\
\hline \multirow[t]{2}{*}{ rs 1333042} & & German & 151 & 736 & Schaefer et al. (2009) [14] \\
\hline & & German $^{\mathrm{b}}$ & 137 & 368 & Schaefer et al. (2009) [14] \\
\hline \multirow[t]{3}{*}{ rs 2891168} & & German & 151 & 736 & Schaefer et al. (2009) [14] \\
\hline & & German $^{\mathrm{b}}$ & 137 & 368 & Schaefer et al. (2009) [14] \\
\hline & & German-Northern Irish & 130 & 339 & Ernst et al. (2010) [15] \\
\hline \multirow[t]{3}{*}{ rs496892 (=rs1292136) } & & German & 151 & 736 & Schaefer et al. (2009) [14] \\
\hline & & German $^{\mathrm{b}}$ & 137 & 368 & Schaefer et al. (2009) [14] \\
\hline & & German-Northern Irish & 130 & 339 & Ernst et al. (2010) [15] \\
\hline $\mathrm{COX} 2$ & $1 \mathrm{q} 25.2-25.3$ & & & & \\
\hline \multirow[t]{2}{*}{ rs20417 (Tag SNP rs6681231) } & & Taiwanese & 85 & 153 & Ho et al. (2008) [17] \\
\hline & & Dutch / German & 520 & 1043 & Schaefer et al. (2010) [18] \\
\hline$G L T 6 D 1^{\mathrm{a}}$ & $9 \mathrm{q} 34.3$ & & & & \\
\hline \multirow[t]{3}{*}{ rs1537415 } & & German & 141 & 500 & Schaefer et al. (2010) [19] \\
\hline & & German $^{\mathrm{b}}$ & 142 & 472 & Schaefer et al. (2010) [19] \\
\hline & & Dutch $^{\mathrm{c}}$ & 155 & 341 & Schaefer et al (2010) [19] \\
\hline
\end{tabular}

${ }^{\mathrm{a}}$ GWA study.

${ }^{\mathrm{b}}$ localized aggressive periodontitis.

${ }^{\mathrm{c}}$ validation in generalized and localized aggressive periodontitis.

It should be noted that these SNPs represent most likely tagging SNPs and not causative variants, but they have identified the genetic region associated with aggressive periodontitis.

suggested evidence for the minor alleles in $I L-1 A$ (rs1800587, also cited in the literature as -889 and in linkage with +4845 ), in $I L-1 B$ (rs 1143634, also cited in the literature as +3953) and for a composite genotype that combines minor alleles at each locus to be risk factors for chronic periodontitis [26-28]. However, the results also demonstrated significant heterogeneity among the included studies.

\section{Genome-Wide Association Studies (GWAS) in Periodontitis}

A genome-wide association study (GWAS) is a powerful molecular technique to analyze hundreds of thousands or even millions of variations in genomic DNA simultaneously and to determine if any locus is associated with a certain disease phenotype.
GWAS analyze SNPs covering the entire human genome with the great majority in regulatory regions and a small part in coding regions. Because of the complex structure of the genome (for example linkage disequilibrium), associated variants are mostly not the causative variants. But the associated variants are expected to be linked with the causative variants. Since in a GWAS an enormous amount of SNPs is determined, it is essential to include large numbers of well-characterized and matched patients and controls, and the statistical significance level is usually set at $<5 \times 10^{-8}$ to correct for extensive multiple testing [29]. Typically, on the basis of a GWAS, novel candidate genetic loci or SNPs may be proposed and the results of a GWAS need to be further validated in an independent cohort with a candidate gene study. Therefore, GWAS are important in hypothesis-generation, seeking for novel candidate genes and pathways, especially in diseases where the genetic basis is not fully understood. 
Today, more than 1300 GWAS have been performed (http://www.genome.gov.gwastudies), but only 4 GWAS have been performed in association with periodontitis; 1 in aggressive periodontitis and 2 in chronic periodontitis $[19,30$, 31]. The fourth GWAS for periodontitis studied SNPs in association with pathologic periodontal pocket depths, instead of in association with a certain form of periodontitis [32]. Furthermore, 1 GWAS examined host genetic risk loci in association with subgingival bacterial colonization [21]. Recently, Rhodin et al. reanalyzed the GWAS data of previous studies $[21,30]$ with a gene-centric and gene set enrichment analyses [33॰]. Although we review the periodontitis GWAS results below, the studies differ in methodology and quality.

\section{Aggressive Periodontitis}

The first periodontitis GWAS was performed in aggressive periodontitis and screened over 1.2 million SNPs [19]. The GWAS discovery cohort consisted of 141 aggressive periodontitis patients and 500 controls, and the second GWAS cohort in the same study consisted of 142 aggressive periodontitis patients and 472 controls, both cohorts with subjects of German origin. From the first GWAS 197 qualitycontrolled SNPs and from the second GWAS 244 SNPs passed the threshold for association. Only the GLT6D1 SNP rs1537415 remained significant in both aggressive periodontitis GWAS cohorts (Table 1). This association was further validated in a Dutch cohort with localized and generalized aggressive periodontitis patients ( 155 cases vs 341 controls) [19]. The GLT6D1 SNP rs1537415 is located within intron 2 of the glycosyltransferase 6 domain containing 1 (GLT6D1) gene. Preliminary functional analyses have shown that the rare allele of SNP rs1537415 results in impaired binding of the transcription factor GATA-3 [19], its functional role in aggressive periodontitis is not clear.

\section{Chronic Periodontitis}

Divaris et al. performed the first GWAS in chronic periodontitis [30]. The study included 761 patients with severe and 1920 with moderate chronic periodontitis, and 1823 periodontally healthy controls of European American origin. No genome-wide significant associations were found $(P<5 \times$ $10^{-8}$ ). However, 6 genetic loci showed suggestive association with chronic periodontitis $\left(P<5 \times 10^{-6}\right)$. In a validation cohort of 686 individuals ( 86 controls, 373 moderate and 197 severe periodontitis patients) 3 of these loci showed concordance with the GWAS results. Moderate chronic periodontitis was associated with SNPs rs7762544 (chromosome 6p21.1, closest gene NCR2) and rs3826782 (chromosome 19p13.3, closest gene EMR1), and severe chronic periodontitis with SNP rs2521634 (chromosome 7p15, closest gene NPY) [30].
Further, their results indicated a role of neurotransmitter and nervous system signaling pathways complementary to immune response-related pathways in chronic periodontitis.

Teumer et al. [31] also used a GWAS to study genetic loci (17 million genetic variations) in association with chronic periodontitis in 2 German populations. The first cohort consisted of 670 severe, 1188 moderate periodontitis patients, and 1507 controls, and the second cohort consisted of 111 severe, 247 moderate periodontitis patients, and 309 controls. No genome-wide significant association was found for chronic periodontitis $\left(P<5 \times 10^{-8}\right)$. However, interestingly, they reported that $34 \%$ of variance for mean approximal periodontal attachment loss in individuals younger than 60 years old, was explained by genetic factors. This is in agreement with the previous reports estimating heritability for mean attachment loss in patients with chronic periodontitis $[2,6]$.

Shaffer et al. [32] performed a GWAS in non-Hispanic Caucasian adults in association with periodontal pocket depth. No significant genome-wide association was found, and in contrast to other GWAS, no discrimination was made between aggressive and chronic periodontitis.

In another approach, subgingival infection patterns with oral bacterial species in chronic periodontitis cases and controls have been investigated as outcome parameter in a GWAS [21]. A total of 1020 Caucasian Americans were included: 416 subjects diagnosed with healthy/mild periodontitis, 415 with moderate, and 189 with severe periodontitis. Subgingival plaque samples were analyzed by DNA-DNA hybridization. Subjects with "high" bacterial colonization of "red" complex species: Pophyromonas gingivalis, Tannerella forsythia, Treponema denticola; "orange" complex species: Prevotella intermedia, Prevotella nigrescens, Fusobacterium nucleatum, Campylobacter rectus, and Aggregatibacter actinomycetemcomitans were compared with the non-"high" colonizers. No genome-wide association with "high" bacterial colonization or chronic periodontitis was found. However, suggestive evidence was found for 13 loci including KCNK1, FBXO38, UHRF2, IL33, RUNX2, TRPS1, CAMTA1 and VAMP3 [21]. These results could not be replicated in an African American cohort $(N=123)$.

The recent gene-centric and gene set enrichment analyses on existing GWAS data have offered a promising approach to identify novel periodontitis genes with less demanding cutoffs for multiple testing [33॰]. Four genes (NIN, $A B H D 12 B$, $W H A M M$, and $A P 3 B 2$ ) were associated with severe chronic periodontitis, and 2 genes (KCNK1 and DAB2IP) with periodontal pathogen colonization. Furthermore, some other genes were proposed for moderate chronic periodontitis. Several of these associations confirmed the suggestive associations of the earlier studies, and need to be replicated in independent cohorts.

Although to date regular GWAS have not identified any genome-wide associations with chronic periodontitis, several suggestive genetic loci have been identified, specifically with 
a gene centric approach $[21,30,31,33 \cdot]$. These results have created several novel candidate genes and will hopefully advance our understanding of genetic factors in pathophysiology of chronic periodontitis. Notably, GWAS have not been able to validate any of previously proposed candidate gene associations; the results of some recent meta-analyses have to be viewed in that light [26-28].

\section{Conclusions}

Currently, 3 susceptibility genes, namely GLT6D1, ANRIL, and $C O X-2$, have repeatedly been associated with periodontitis [13-19]. Most of the associations have been reported in the aggressive form of periodontitis emphasizing the importance of genetic factors in biology of this severe periodontitis phenotype. Interestingly, polymorphisms within ANRIL and COX-2 tended to be associated also with chronic periodontitis $[13,17,24,25]$. Further, SNPs within the regulatory regions of the $I L-10$ gene have shown suggestive associations with aggressive periodontitis, and SNPs in DEFB1 with both aggressive and chronic periodontitis [16, 23]. Probably, these SNPs are "genetic markers" and not causative variants for aggressive and chronic periodontitis.

The identified genetic risk variants for periodontitis are all located within introns (ANRIL and GLT6D1) or regulatory regions (COX-2, IL-10, and DEFB1) [34•]. This is in line with other complex diseases; the majority of common human genomic variants that are genome-wide associated with over 400 complex diseases and traits are located within regulatory and intronic regions and not within coding regions [34•]. Genetic variations of intronic and regulatory regions may lead to subtle changes in the expression of associated coding regions and may affect the quantity of the transcription and subsequent protein product.

Thus far, GWAS have had limited success in identifying genetic markers in periodontitis and surprisingly, they have not been able to corroborate any of candidate gene associations. Only GLT6D1 SNP rs1537415 has been identified with GWAS in aggressive periodontitis [19] and no significant genome-wide associations have been found in chronic periodontitis. This may be due to the milder disease phenotype of chronic periodontitis, the applied conservative significance threshold $\left(\mathrm{P}<5 \times 10^{-8}\right)$, and the power of the GWAS in chronic periodontitis to detect significant genetic markers may be too low given the relative small sample sizes of these studies.

Many complex diseases, such as rheumatoid arthritis, Crohn's disease, type 2 diabetes, cardiovascular diseases, and other inflammatory diseases share genetic risk factors [8]. Pleiotropy has been found for $13.2 \%-18.6 \%$ of disease associated genes and in $4.6 \%-7.8 \%$ of SNPs associated with disease or disease traits (http://www.genome.gov). The sharing of genetic variants for multiple diseases and phenotypes may generate new candidate genes for other diseases and can also be incorporated into the future research on genetic risk variants in aggressive and chronic periodontitis [34•]. However, not all genetic variants are shared between inflammatory diseases or not even between the aggressive and chronic periodontitis. For example, the genome-wide association of GLT6D1 with aggressive periodontitis has not been found in any other genetic disease or trait not even in chronic periodontitis [34•].

All individuals harbor millions of genetic variants (SNPs, insertions, deletions, repeats, etc.). The majority of these variants most likely have no effect, while the minority may have some function, mainly physiological and not pathologic, and are mainly responsible for the "normal" phenotypic differences between individuals. However, some combinations of the inherited variants can make individuals susceptible for certain traits or diseases, in particular in combination with unfavorable environmental and lifestyle factors; in particular if the latter are unfavorable for the host resistance, the genetic penetrance of inherited variants increases [35]. For periodontitis, we are searching for the genetic risk factors to better understand the disease and to be able to identify pathobiological pathways in which genes, environment and lifestyle are interconnected. Future studies need to concentrate on larger sample sizes in multiple ethnic populations and to use mathematical modeling (for example [36•]) of the various causative factors to further explore the complexity of periodontitis.

\section{Compliance with Ethics Guidelines}

Conflict of Interest Soren Jepsen is supported in part by a grant from the German Research Foundation (DFG). Bruno G. Loos has received a grant from the Dutch Society of Periodontology for experimental work related to the subject of the role of genetics in the etiology of periodontitis. This current review is not specifically supported by any grant money.

Marja L. Laine and Bruno G. Loos are supported in part by a grant from the University of Amsterdam, the Netherlands, for the focal point "Oral infection and inflammation."

Human and Animal Rights and Informed Consent No human or animal studies performed by the authors. This article does not contain any studies with human or animal subjects performed by any of the authors.

\section{References}

Papers of particular interest, published recently, have been highlighted as:

- Of importance

1. Van der Velden U, Abbas F, Armand S, et al. Java project on periodontal diseases. The natural development of periodontitis: risk factors, risk predictors and risk determinants. J Clin Periodontol. 2006;33:540-8. 
2. Michalowicz BS, Aeppli D, et al. Periodontal findings in adult twins. J Periodontol. 1991;62:293-9.

3. Marazita ML, Burmeister JA, Gunsolley JC, Koerge JC, Lake K, Schenkein HA. Evidence for autosomal dominant inheritance and race-specific heterogeneity in early-onset periodontitis. J Periodontol. 1994;65:623-30.

4. Laine ML, Loos BG, Crielaard W. Gene polymorphisms in chronic periodontitis. Int J Dent. 2010;324719.

5. Ciancio SG, Hazen SP, Cunat JJ. Periodontal observations in twins. J Periodontal Res. 1969;4:42-5.

6. Mucci LA, Bjorkman L, Douglass CW, Pedersen NL. Environmental and heritable factors in the etiology of oral diseases - a population-based study of Swedish twins. J Dent Res. 2005;84:800-5.

7. Stabholz A, Soskolne WA, Shapira L. Genetic and environmental risk factors for chronic periodontitis and aggressive periodontitis. Periodontology 2000. 2010;53:138-53.

8. Sivakumaran S, Agakov F, Theodoratou E, et al. Abundant pleiotropy in human complex diseases and traits. Am J Hum Genet. 2011;89:607-18.

9. Ioannidis JP. Genetic associations: false or true? Trends Mol Med. 2003;9:135-8.

10. Laine ML, Crielaard W, Loos BW. Genetic susceptibility to periodontitis. Periodontology 2000. 2012;58:37-68.

11. Zhang J, Sun X, Xiao L, Xie C, Xuan D, Luo G. Gene polymorphisms and periodontitis. Periodontology 2000. 2011;56:102-24.

12. Schaefer AS, Jepsen S, Loos BG. Periodontal genetics: a decade of genetic association studies mandates better study designs. J Clin Periodontol. 2011;38:103-7.

13. Schaefer AS, Bochenek G, Manke T, et al. CDKN2BAS is associated with periodontitis in different European populations and is activated by bacterial infection. J Med Genet. 2011;48:38-47.

14. Schaefer AS, Richter GM, Groessner-Schreiber B, et al. Identification of a shared genetic susceptibility locus for coronary heart disease and periodontitis. PLoS Genet. 2009;5:e1000378.

15. Ernst FD, Uhr K, Teumer A, et al. Replication of the association of chromosomal region 9p21.3 with generalized aggressive periodontitis ( $\mathrm{gAgP}$ ) using an independent case-control cohort. BMC Med Genet. 2010;11:119.

16. Schaefer AS, Bochenek G, Manke T, et al. Validation of reported genetic risk factors for periodontitis in a large-scale replication study. J Clin Periodontol. 2013;40:563-72.

17. Ho YP, Lin YC, Yang YH, Ho KY, Wu YM, Tsai CC. Cyclooxygenase-2 Gene-765 single nucleotide polymorphism as a protective factor against periodontitis in Taiwanese. J Clin Periodontol. 2008;35:1-8.

18. Schaefer AS, Richter GM, Nothnagel M, et al. COX-2 is associated with periodontitis in Europeans. J Dent Res. 2010;89:384-8.

19. Schaefer AS, Richter GM, Nothnagel M, et al. A genome-wide association study identifies GLT6D1 as a susceptibility locus for periodontitis. Hum Mol Genet. 2010;19:553-62.

20. Bochenek G, Hasler R, El Mokhtari NE, et al. The large non-coding RNA ANRIL, which is associated with atherosclerosis, periodontitis and several forms of cancer, regulates ADIPOR1, VAMP3 and C11ORF10. Hum Mol Genet. 2013;22:4516-27.
21. Divaris K, Monda KL, North KE, et al. Genome-wide association study of periodontal pathogen colonization. J Dent Res. 2012;91(7 Suppl):21S-8.

22. Lees CW, Barrett JC, Parkes M, Satsangi J. New IBD genetics: common pathways with other diseases. Gut. 2011;60:1739-53. doi: 10.1136/gut.2009.199679.

23. Schaefer AS, Richter GM, Nothnagel M, et al. A 3' UTR transition within DEFB1 is associated with chronic and aggressive periodontitis. Genes Immun. 2010;11:45-54.

24. Loo WT, Wang M, Jin LJ, Cheung MN, Li GR. Association of matrix metalloproteinase (MMP-1, MMP-3 and MMP-9) and cyclooxygenase- 2 gene polymorphisms and their proteins with chronic periodontitis. Arch Oral Biol. 2011;56:1081-90.

25. Li G, Yue Y, Tian Y, et al. Association of matrix metalloproteinase (MMP)-1, 3, 9, interleukin (IL)-2, 8 and cyclooxygenase (COX)-2 gene polymorphisms with chronic periodontitis in a Chinese population. Cytokine. 2012;60:552-60.

26. Deng JS, Qin P, Li XX, Du YH. Association between interleukin1beta C (3953/4)T polymorphism and chronic periodontitis: evidence from a meta-analysis. Hum Immunol. 2013;74:371-8.

27. Karimbux NY, Saraiya VM, Elangovan S, et al. Interleukin-1 gene polymorphisms and chronic periodontitis in adult whites: a systematic review and meta-analysis. J Periodontol. 2012;83:1407-19.

28. Mao M, Zeng XT, Ma T, He W, Zhang C, Zhou J. Interleukin1alpha $-899(+4845) \mathrm{C}->\mathrm{T}$ polymorphism increases the risk of chronic periodontitis: evidence from a meta-analysis of 23 casecontrol studies. Gene. 2013;532:114-9.

29. Manolio TA. Genomewide association studies and assessment of the risk of disease. N Engl J Med. 2010;363:166-76.

30. Divaris K, Monda KL, North KE, et al. Exploring the genetic basis of chronic periodontitis: a genome-wide association study. Hum Mol Genet. 2013;22:2312-24.

31. Teumer A, Holtfreter B, Volker U, et al. Genome-wide association study of chronic periodontitis in a general German population. J Clin Periodontol. 2013;40:977-85.

32. Shaffer JR, Polk DE, Wang X, et al. Genome-wide association study of periodontal health measured by probing depth in adults ages 18-49 years. G3 (Bethesda). 2014;4:307-14.

33. Rhodin K, Divaris K, North KE, et al. Chronic periodontitis genome-wide association studies: gene-centric and gene set enrichment analyses. J Dent Res. 2014;93:882-90. This article reports on an approach to identify novel periodontitis genes with less demanding cutoffs for multiple testing.

34. Vaithilingam RD, Safii SH, Baharuddin NA, et al. Moving into a new era of periodontal genetic studies: relevance of large case-control samples using severe phenotypes for genome-wide association studies. J Periodontal Res. 2014. doi:10.1111/jre.12167. This review discusses the current problems faced in periodontal genetic studies and lessons to be learned from previous periodontal genetic studies.

35. Manolio TA, Collins FS, Cox NJ, et al. Finding the missing heritability of complex diseases. Nature. 2009;461:747-53.

36. Laine ML, Moustakis V, Koumakis L, Potamias G, Loos BG. Modeling susceptibility to periodontitis. J Dent Res. 2013;92:45-50. This article shows that on basis of genetic and microbial factors patterns may be recognized to identify patients at risk for periodontitis. 